\title{
GEOMETRIC SURFACE MODIFICATION OF NOZZLES FOR COMPLETE TRANSFER OF LIQUID DROPS
}

\author{
Ui-Chong Yi and Chang-Jin "CJ" Kim \\ Mechanical and Aerospace Engineering Dept., University of California, Los Angeles (UCLA) \\ Los Angeles, California 90095
}

\begin{abstract}
We report a new and unique way of promoting the complete transfer of discrete liquid drops from a nozzle to printing surfaces when the transfer (i.e., printing) is made by the wettability differences between two surfaces as used in soft printing devices reported previously. Hypothesizing that the complete transfer of droplets can be promoted by reducing the surface energy at the nozzle surface, we propose a new nozzle design with serrated circumference. By geometrically limiting the liquid-solid contact area on the nozzle surface, the effective energy holding droplets back can be reduced. Tests of droplet transfers $(\sim 1 \mu$ in volume) from hydrophobic nozzles $(\sim 500 \mu \mathrm{m}$ in diameter) to hydrophilic printing surfaces (e.g., glass surfaces) confirm that the residual droplets can be eliminated and perfect transfer can be accomplished when the nozzles are made serrated.
\end{abstract}

\section{INTRODUCTION}

Printing microscopic liquid droplets onto solid surfaces has become an important scientific/engineering issue in recent years, in demand by such major areas as inkjet printing and DNA/protein microarrays. For microarrays, the amounts of target molecules are determined by the droplet volumes printed on each spot; the variations in the droplet volume would cause an error in the detection of genes or gene expressions. The printing of droplets with consistent volume is an important issue.

Soft printing (i.e., droplet transfer through liquid-solid contact only) has been developed to avoid the tip deformation in pin-based printing and splashing in inkjetbased printing [1]. It is worth noting that the concept of soft printing assumes liquid is available as discrete droplets prior to printing by such mechanism as electrowetting on dielectric (EWOD). While consistent volumes of droplets were generated in the printhead, this consistency was found lost after printing, unfortunately, because each printing action leaves a small residual volume behind at the nozzle [1]. It is the main goal of this paper to eliminate the residual droplets and restore the volume consistency of the printed droplets.

By promoting complete transfers of discrete drops without residuals on nozzles, different samples can be printed through one nozzle, enabling a flexible and compact system. It also speeds up the printing processes by minimizing the cleaning steps typically employed to avoid cross contamination among printed spots. Further, the concept has possible applications to other areas such as inkjet printings (for elimination of satellite droplets) and DNA solid pin "Replicator" (for reducing the need for cleaning). As droplet-based microfluidics become widely accepted, the complete transfer of liquid droplets will play an important role.

We developed a simple and effective method for the complete transfers of liquid droplets from a printhead to the printing surfaces by micro-engineering the inside circumference of the nozzle (Figure 1) based on the concept of minimizing effective surface energy $[2,3]$. By generating artificial roughness on hydrophobic surfaces, the effective liquid-solid surface energy can be reduced far beyond the intrinsic material property allowing a dramatic drag reduction $(>95 \%)$ for moving droplets inside a channel [3]. We apply a similar idea to the transfer of droplets from printheads to print surfaces by soft printing. Soft printing uses hydrophobic printheads and hydrophilic printing surfaces (e.g., glass plate) so that the droplets favor the printing surfaces when they are pulled apart after the soft contact. By reducing the effective energy on the nozzle circumference with serrated structures thus minimizing the pull back of the printhead during soft printing, we promote a complete transfer with no new cost.

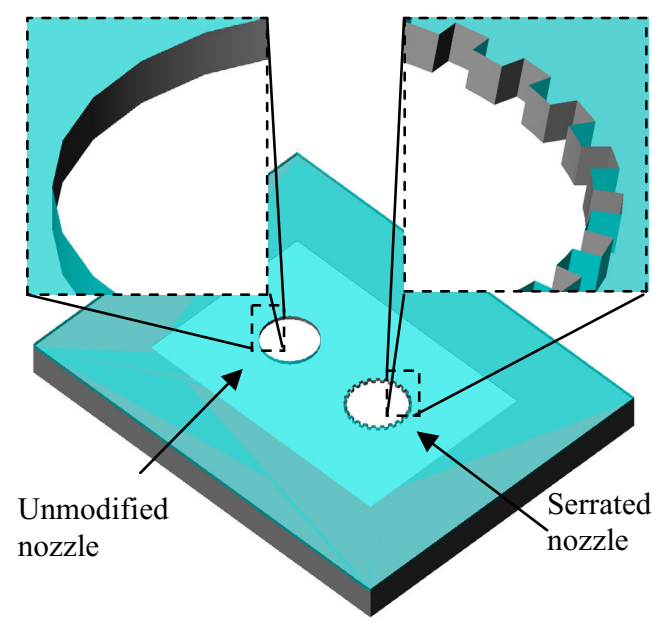

Figure 1. Unmodified and serrated nozzles

\section{THEORY}

Figure 2 illustrates an analytical model for droplet separation during transfer. The figure only illustrates the printing steps after soft contact between liquid from the nozzle and the top hydrophilic printing surface for simplicity. Chadov et al. [4] had performed a similar analysis to study 
droplet behaviors during the transfer between two solid surfaces. In the study, he predicted the volume ratio of separated drops based on droplet volumes and surface properties.

Once the contact between the liquid and the plate is made, the hydrophilic top plate moves away from the hydrophobic nozzle, and the droplet necking occurs to preserve the volume of the droplet. Contact angle $\theta$ measured from the side surface of the nozzle to the liquid meniscus must be reduced simultaneously as seen in figure 2. As the necking progresses, the air-liquid interfaces at that region finally meet at the center, separating the liquid into two drops at a corresponding separation contact angle $\theta_{\text {sep }}$.

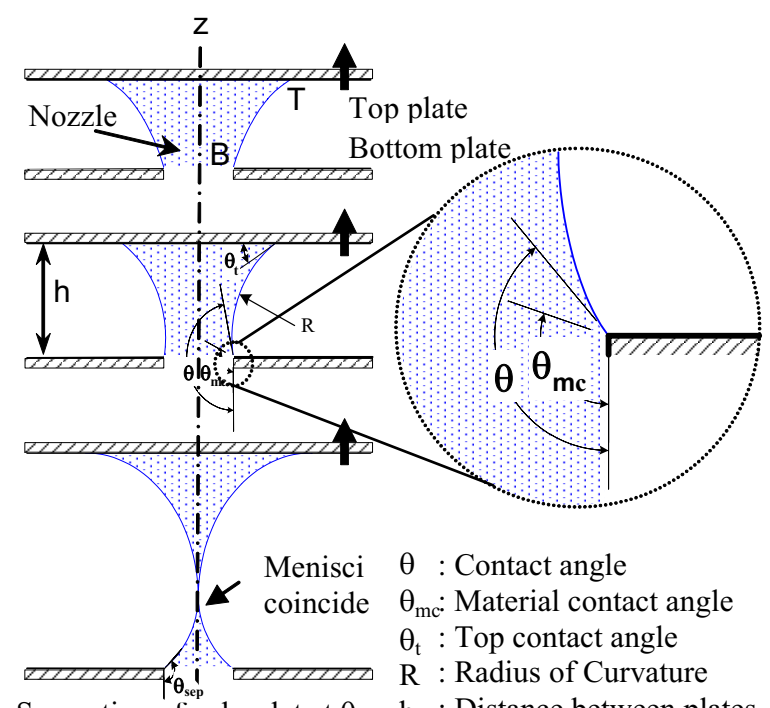

Separation of a droplet at $\theta_{\text {sep }} \mathrm{h}$ : Distance between plates

Figure 2. Analytical model for separation during transfer

Assuming the droplet shape is approximately formed by the rotation of the circular arc $T B$ with radius of curvature $R$ around the $z$ axis, the separation can be predicted by calculating $\theta_{\text {sep }}$ for a given drop volume and surface properties of the nozzle and the printing plate as well as dimensions. The calculated separation angles of droplets are plotted against droplet volumes normalized with the nozzle radius in figure 3 . For the calculation of separation contact angles, the droplet volume was assumed to be constant, and no effect of contact angle hysteresis was considered.

For a liquid drop to be separated into two smaller drops, contact angle $\theta$ must reach the value of the separation contact angle $\theta_{\text {sep }}$. However, the contact angle can not be reduced beyond the value of the intrinsic material contact angle $\theta_{m c}$ of the nozzle side surface. If $\theta_{s e p}$ is smaller than the value of $\theta_{m c}$ for a given volume of liquid and surfaces, the top plate will continue to pull the liquid without separation until the transfer is complete. By increasing the material contact angle $\theta_{m c}$ (i.e., minimizing effective surface energy) through geometrically modifying the surface as shown in figure 1, we propose complete transfers of droplets can be achieved. A droplet on a nozzle (i.e., normalized volume $V^{*}=64$ ) would be separated into two drops with $\theta_{m c}$ less than $\theta_{\text {sep }}\left(=133^{\circ}\right)$ for the given volume (Figure 3). For example, a $1 \mu \mathrm{l}$ droplet on a nozzle with $500 \mu \mathrm{m}$ in diameter would be separated when printed through a regular (i.e., unmodified) nozzle coated with $\operatorname{Cytop}^{\circledR}\left(\theta_{m c}=105^{\circ}\right)$. However, if the surface contact angle is increased to over $133^{\circ}$, the drop will be transferred to the top plate without any residual drop on the nozzle side.

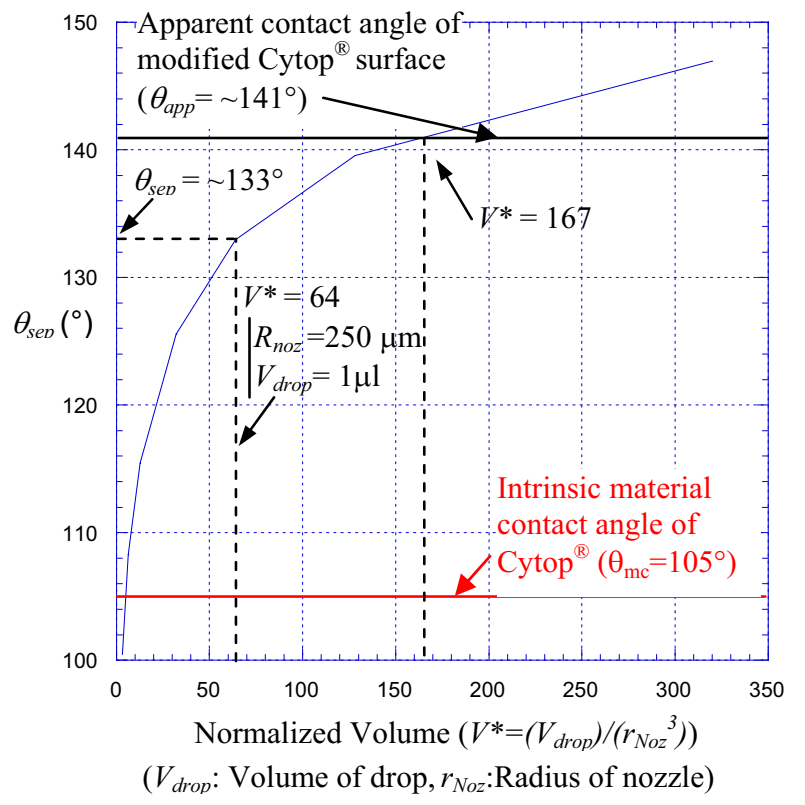

Figure 3. Angle of separation with contact angle of intrinsic and modified surface of Cytop ${ }^{\circledR}$

The apparent contact angle on a roughened surface is expressed by Cassie's equation [5]

$$
\cos \theta_{\text {app }}=f_{1} \cdot \cos \theta_{m c}-f_{2}
$$

where $\cos \theta_{a p p}$ is the apparent contact angle on the modified surface and $\cos \theta_{m c}$ is the intrinsic material contact angle. $f_{l}$ and $f_{2}$ are the area fractions of liquid-solid and liquid-air contacts. As can be seen from the equation, the reduction in $f_{l}$ leads to the increase in the surface contact angle and can be used for promoting complete liquid transfers.

\section{DESIGN AND FABRICATION}

The samples are designed (Figure 4) and fabricated through micromachining based on the calculations from the above criteria. Three degrees of nozzle modifications are included with size variations in serrated structures to evaluate the performance. For the variations in structures, the lengths of the liquid-solid contact area (i.e., $f 1$ in figure 4) are designed to be $2 \mu \mathrm{m}, 5 \mu \mathrm{m}$, and $16 \mu \mathrm{m}$ to test size effect on liquid transfers. Unmodified, $1 / 4$ serrated, 1/2 serrated, and fully serrated nozzles are designed to determine the minimum modification required to promote the complete transfers. In all the designs, the size of the nozzle is set to be $500 \mu \mathrm{m}$ in diameter and the area reduction of the solid-liquid area is about $70 \%$ compared with the unmodified surface. $70 \%$ reduction in solid-liquid contact 
area results in the increase of the contact angle of hydrophobic side surface of the nozzle (i.e., Cytop ${ }^{\circledR}$-coated surface) to $\theta_{a p p}$ of $\sim 141^{\circ}$ from $\theta_{m c}$ of $\sim 105^{\circ}$ according to equation 1. With the increased $\theta_{\text {app }}$, a droplet with $V^{*}<$ $\sim 167$ would completely transfer to the printing surface, according to the criteria. The results of the calculations for the designs are co-plotted in figure 3 with separation contact angles.

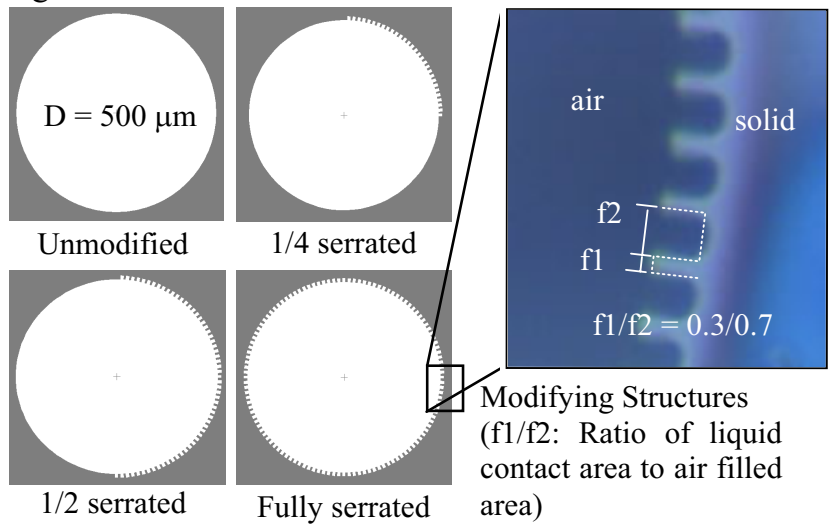

Figure 4. Four types of nozzles (different degrees of modification around nozzle) and fabricated serrated structures

Figure 5 illustrates the schematic cross-sectional view of a fabricated testing device, micromachined from a 100 $\mu$ m-thick silicon wafer. The wafer was deposited with thermal silicon dioxide $\left(\mathrm{SiO}_{2}\right)$ and LPCVD silicon nitride $\left(\mathrm{Si}_{3} \mathrm{~N}_{4}\right)$ layers to be used as protection layers against $\mathrm{KOH}$ etching. A $2 \mu \mathrm{m}$-thick low stress nitride (LSN) layer was deposited on the top side as a structural top layer. The top and bottom side of the wafer are then etched by RIE to form nozzles. The top side LSN layer was carefully patterned to form nozzles with their corresponding serrated circumferences. The bottom side $\mathrm{SiO}_{2}$ and $\mathrm{Si}_{3} \mathrm{~N}_{4}$ layers were also patterned to form the LSN membranes later. The patterned wafer went through $\mathrm{KOH}$ silicon etching step to form LSN membrane, which works as a structural transparent top layer. The device was then spin coated with ample amounts of Cytop ${ }^{\circledR}$ solution, which was chosen for its better wetting property on nitride surface over Teflon ${ }^{\circledR}$. A bottom glass plate is also coated with Cytop $^{\circledR}$ to be used as a bottom channel surface.

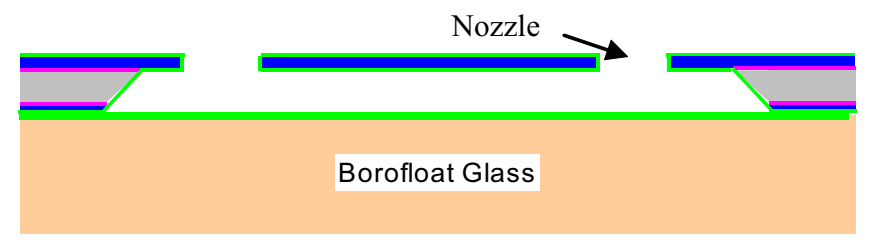

Cytop $^{\circledR}$ layer - hydrophobic coating

$100 \mu \mathrm{m}$ thick Si wafer $\quad \mathrm{SiO}_{2} / \mathrm{Si}_{3} \mathrm{~N}_{4}$ layer

$\sim 2 \mu \mathrm{m}$ thick low stress nitride layer (LSN)

Figure 5. Cross section view of micromachined printhead test device

\section{TESTS AND RESULTS}

The procedures for testing are illustrated in Figure 6. A small volume $(\sim 1 \mu \mathrm{l})$ of liquid is pipetted on a Cytop ${ }^{\circledR}$ coated glass substrate surface, the LSN membrane plate is placed over it and manually pushed down to have the droplet bulge up through the nozzle. The top membrane plate and the bottom substrate plate are then temporarily bonded with $3 \mathrm{M}^{\circledR}$ double-stick tapes during the remaining printing test steps. A glass printing plate is then lowered to make the soft contact with the liquid and then moved away, completing one printing cycle. A microscope was used for the visualization and recording of the test from the side.

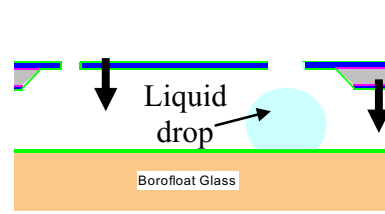

(a)

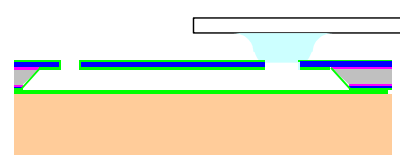

(c)

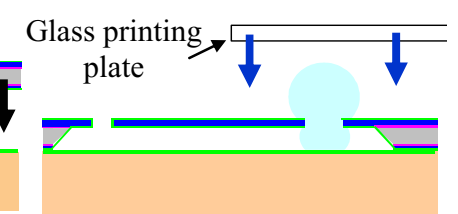

(b) 4
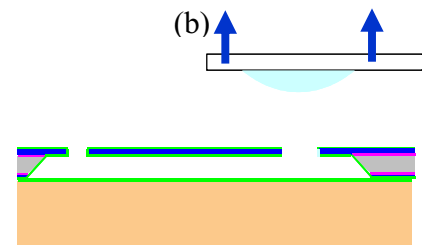

(d)
Figure 6. Printing test. (a) Loading liquid and positioning the membrane plate down (b) Moving a glass plate down (c) Establishing liquid-solid contact (d) Pulling the glass plate away and completing the transfer

Figure 7 illustrates the result of droplet transfer tests done with water. While the unmodified nozzle leaves residual water behind, all three types of serrated nozzles show complete transfers onto the glass plates. The

(0)

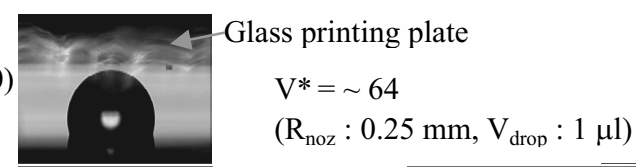

(1)

(2)

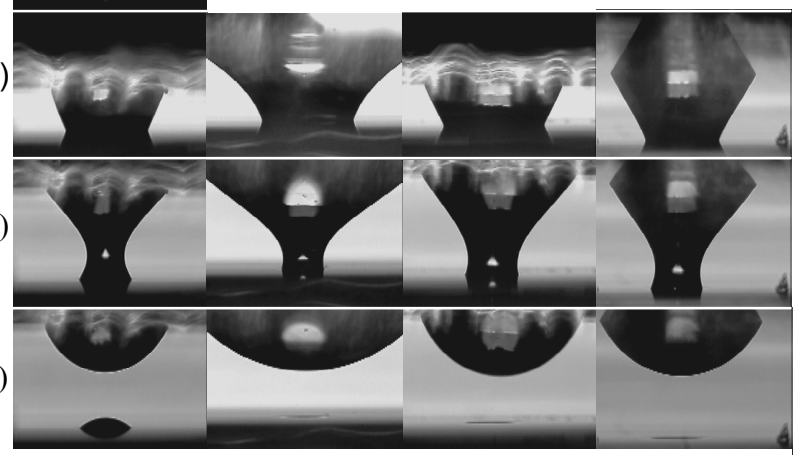

Unmodified $\quad 1 / 4$ serrated $\quad 1 / 2$ serrated Fully serrated

Figure 7. Water printing tests with 4 different nozzle types (0) before contact (1) after initial contact (2) during top glass plate pulling away (3) after transfer of droplet 
normalized volumes of the droplets were attempted to be at 64 during the tests. The exact detaching moment could not be captured with our current camera (60 frames/sec.). We did not observe any apparent effect of the size variations for the transfers; nozzles with the same design with different dimensions of serrated structures showed the same results. We conclude that the ratio of solid-liquid contact area to liquid-air contact area is the dominant factor.

The nozzles were also tested with a DNA solution (Calf Thymus, $4 \mu \mathrm{g} / \mathrm{ml}$ ) successfully (Figure 8 ). The test results verified that complete transfers are obtained if at least $1 / 2$ of the nozzle circumference is modified, leaving rooms of success for even more challenging solutions with lower intrinsic material contact angles.

(1)

(2)

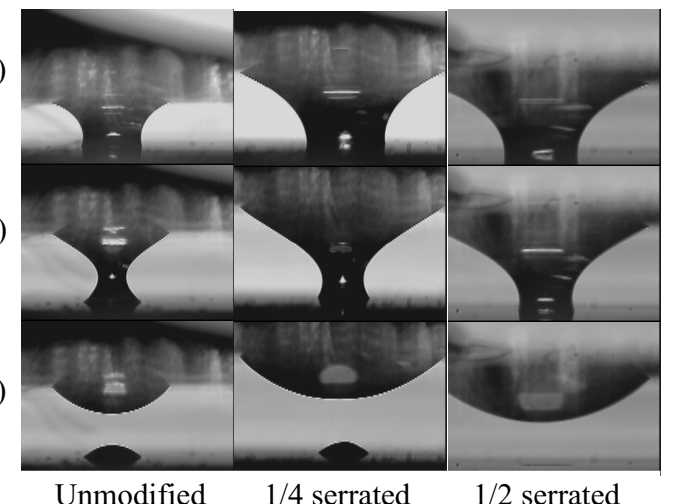

Figure 8. DNA solution tests with 3 different nozzle types (1) after initial contact (2) during top glass plate pulling away (3) after transfer

\section{DISCUSSIONS AND FUTURE WORK}

We have not performed any measurement or characterization on the sizes of printed spots since the proposed concept utilizes small discrete droplets readily available prior to actual transferring process. The consistency of the printed spots would be achieved if uniformly sized droplets are delivered to the nozzles and whole droplets are printed without residuals. As used in soft printing, EWOD is inherently capable of generating and transporting small and consistent discrete droplets and the proposed concept of serrated nozzles can be easily integrated with little difficulties. The characterization of the printed spots will be pursued in the future once an integrated device is available for testing.

During the transfer tests with diluted DNA solutions, we have observed that a higher degree of modification is needed to achieve the complete transfer compared with DI water. The need for more modification is suspected to be from the differences in solution properties, such as the contact angle. In our previous contact angle measurement of a diluted DNA solution on Cytop ${ }^{\circledR}$ coated surface, $a \sim 5$ to $10^{\circ}$ lower contact angle was observed while the contact angle for DI water was $\sim 105^{\circ}$. The properties of liquids to be printed are one of the major considerations for determining the design of nozzles and need to be studied further. More tests, including contact angle measurements and transferring tests, with various biological solutions will be performed in the future.

\section{CONCLUSIONS}

The concept of the complete transfer of a liquid droplet by modifying printing nozzles has been proposed and demonstrated with micromachined testing devices. The reduction of effective interfacial energy and its effect on promoting total transfers were analytically predicted and modeled as a function of the surface geometry and the contact angle for the design of nozzles. We have successfully verified the concept by demonstrating the total transfers of water and diluted DNA solution droplets onto the glass plates. We expect the technology to be useful for microfluidic printing systems, especially those employing small discrete droplets.

\section{ACKNOWLEDGEMENTS}

This work was supported by the National Science Foundation (NSF) "Engineering Microsystems: XYZ on a Chip" (CMS-99-80874) and NASA through the Instritue for Cell Mimetic Space Exploration (CMISE). The author would like to thank Dr. J.-Y. Yoon for the preparation of DNA solutions.

\section{REFERENCES}

1. U.-C. Yi and C.-J. Kim, "Soft Printing of Droplets Digitized by Electrowetting", Digest of Technical Papers of the Transducers 2003, Boston, MA, 6/8-6/12, (2003) pp. 1239-1242.

2. D. Oner and T. J. McCarthy, "Ultrahydrophobic Surfaces. Effect of Topography Length Scales on Wettability", Langmuir, 16, pp. 7777-7782 (2000).

3. J. Kim and C.-J Kim, "Nanostructured Surfaces for Dramatic Reduction of Flow Resistance in Droplet-based Microfluidics", Proceedings of the IEEE Conference on MEMS, Las Vegas, NV, 1/20-1/24, (2002), pp. 479-482.

4. A. V. Chadov and E. D. Yakhnin, "Investigation of the Transfer of a Liquid from One Solid Surface to Another. I. slow Transfer, Method of Approximate Calculation", Kolloidnyi Zhurnal, 41, 4, pp. 817-820 (1979).

5. A. W. Adamson, "Physical Chemistry of Surfaces, $5^{\text {th }}$ edition", John Wilely \& Sons, Inc., New York, NY (1990), pp. 387-389. 